\section{PROFILES OF BENIGN POSITIONAL VERTIGO TESTED ON THE EPLEY OMNIAX CHAIR}

${ }^{1,2}$ Emma C Argaet $^{*},{ }^{1,2}$ Corinna Lechner, ${ }^{1,2}$ Andrew P Bradshaw, ${ }^{1,2} \mathrm{G}$ Michael Halmagyi, 1,2 Miriam S Welgampola. 'Institute of Clinical Neurosciences, Royal Prince Alfred Hospital, Sydney, NSW, Australia; ' Central Clinical School, University of Sydney, Sydney, NSW, Australia

\subsection{6/jnnp-2019-anzan.20}

Introduction Benign positional vertigo (BPV) has a characteristic pattern of nystagmus specific to the affected semicircular canal and the underlying mechanism of canalithiasis (where otoconia float freely) or cupulolithiasis (where otoconia are adherent to the cupula).

Methods We analysed the nystagmus slow-phase velocity (SPV) profiles of 100 subjects with posterior-canalithiasis, 30 with lateral-canalithiasis, 10 with lateral-cupulolithiasis and 3 with anterior-canalithiasis. Subjects were examined on the Epley Omniax Rotator, a mechanical chair with real-time video-oculography. Video data was analysed using custom-made LabVIEW software. Nystagmus onset, duration, peak-velocity, peak-latency and time taken for the peak-velocity to halve (t50), were measured.

Results In posterior-canalithiasis, nystagmus occurred within 14.2 seconds of positioning and lasted 2.5-34.5 seconds. The median vertical peak-SPV was $37.3 \%$. The median peaklatency was 2.9 seconds and the median t50 was $3.4 \mathrm{sec}-$ onds. In lateral-canalithiasis, nystagmus onset was mostly immediate. With the affected ear down, the median peakSPV was $52.2 \%$ and the median peak-latency was $3.6 \mathrm{sec}-$ onds; the t50 was 7 seconds (median) and the paroxysms lasted 9.9-48.5 seconds. In lateral-cupulolithiasis, nystagmus onset was instantaneous. With the unaffected ear down, the median peak-SPV was $69.6 \%$ s. The peak-latency (median 18.6 s) and t50 (median $34.5 \mathrm{~s}$ ) were significantly prolonged compared to canalithiasis. For anterior-canalithiasis, the onset was $0-2.9$ seconds, the peak-latency was $3-5.4$ seconds, the t50 was 6.4-10.5 seconds and the duration was 13.4-23.1 seconds.

Conclusions Canalithiasis and cupulolithiasis produce distinct SPV profiles, which enable their identification and the separation of BPV from other causes of positional nystagmus.

\section{PATIENT-INITIATED EVENT MONITORING FOR ACUTE VERTIGO}

${ }^{1}$ Allison S Young*, ${ }^{1}$ Corinna Lechner, ${ }^{1}$ Andrew P Bradshaw, ${ }^{2}$ Hamish G MacDougall, ${ }^{3}$ Deborah A Black, 'Michael G Halmagyi, 'Miriam S Welgampola. 'School of Medicine, University of Sydney, Camperdown, NSW, Australia; ${ }^{2}$ School of Psychology, University of Sydney, Camperdown, NSW, Australia; ${ }^{3}$ Faculty of Health Sciences, University of Sydney, Camperdown, NSW, Australia

\subsection{6/jnnp-2019-anzan.21}

Introduction The diagnosis of vestibular disorders may be facilitated by analysing patient-initiated capture of ictal nystagmus.

Methods Adults with a history of recurrent vertigo were taught to self-record spontaneous and positional-nystagmus at home while symptomatic, using video-goggles. Patients with final diagnoses of disorders presenting with recurrent vertigo were analysed: 121 patients with Ménière's Disease (MD), Vestibular Migraine (VM), Benign Positional Vertigo (BPV), Episodic Ataxia Type II (EAII), Vestibular Paroxysmia (VP) or
Superior Semicircular Canal Dehiscence (SSCD) were included.

Results Of 43 MD patients, 40 showed high-velocity spontaneous horizontal-nystagmus (median slow-phase velocity (SPV) 39.7 degrees/second ( $\%$ s); Twenty-one showed horizontal-nystagmus reversing direction within 12-hours (24 on separate days). In 44 of 67 patients with VM, low velocity spontaneous horizontal $(n=28,4.9 \%$ s), up-beating $(n=6,15.5 \%$ s) or down-beating-nystagmus $(\mathrm{n}=10,5.1 \%$ s) was observed; Sixteen showed positional-nystagmus only, and seven had no nystagmus. Spontaneous horizontal-nystagmus with SPV $>12.05 \%$ had a sensitivity and specificity of $95.3 \%$ and $82.1 \%$ for MD. Nystagmus direction-change within 12-hours was highly specific (95.7\%) for MD. Spontaneous vertical-nystagmus was highly specific (93.0\%) for VM. In the seven BPV patients, spontaneous-nystagmus was absent or $<3 \%$, and characteristic paroxysmal positional nystagmus was observed in all cases. Patients with central and MD-related positional vertigo demonstrated persistent nystagmus. Two patients with EAII showed spontaneous vertical nystagmus, one patient with VP showed short bursts of horizontal-torsional nystagmus lasting 5-10s, and one patient with SSCD demonstrated paroxysmal torsional down-beating nystagmus when supine.

Conclusions Patient-initiated vestibular event-monitoring is feasible and could facilitate rapid and accurate diagnosis of episodic vestibular disorders.

\section{GENETIC CARRIER SCREENING FOR DUCHENNE MUSCULAR DYSTROPHY: THE OUTCOME OF OVER TWENTY YEARS OF GENETIC COUNSELLING ON DISEASE EPIDEMIOLOGY IN A SINGLE-CENTRE COHORT STUDY IN NEW SOUTH WALES (NSW), AUSTRALIA}

${ }^{1}$ Didu Sanduni Kariyawasam*, 'Hugo Samapio, 'David Mowat, ${ }^{1,2}$ Michelle Farrar. 'Sydney Children's Hospital, Randwick, NSW, Australia; ' University of New South Wales, Associate Professor, Kensington, NSW, Australia

\subsection{6/jnnp-2019-anzan.22}

Introduction Duchenne Muscular Dystrophy (DMD), an Xlinked recessive genetic disorder is maternally inherited in approximately two-thirds of affected boys. Female relatives have carrier risk. This study proposes that proactive genetic screening and counselling for patients' relatives, contributes to reductions in preventable cases and ultimately disease incidence.

Methods A retrospective study of cases born in NSW from 1991-2013 was completed, using an electronic database of live male and prenatally diagnosed patients with DMD, referred to our tertiary service. Proband genotype/phenotype, pedigree, carrier-risk and extent of cascade screening were reviewed. Variance analysis (two-way ANOVA) was used to analyse changing trends in preventable cases.

Results 77 cases were identified. Mean age at presentation fell by 14-months over time. Probands were defined as 'theoretically preventable' when disease was identified in previous generations, or in males aged over 6 years, within the same generation. Fifteen (19\%) cases were preventable, with a statistically significant decline in such cases over time.

Cascade screening and prenatal testing of subsequent pregnancies was offered to all carrier mothers and female relatives in the mother's generation. Fifteen women underwent prenatal testing. Three affected male foetuses were identified, with one 
live affected male being born, after parents proceeded with the pregnancy.

Conclusion Rigorous and expansive approaches to cascadescreening/counselling may account for decreases in preventable cases. Age of diagnosis has fallen, providing potential for more timely intervention, aided by newer diagnostic techniques that allow more accurate proband genotyping. Prenatal testing identified small numbers of affected males, facilitating parental decision-making in these select cases.

\section{RESISTANCE EXERCISES WITH BLOOD FLOW RESTRICTION IN PATIENTS WITH SPORADIC INCLUSION BODY MYOSITIS}

${ }^{1}$ Christina Liang*, 'Melanie Burk, ${ }^{1}$ Abby Wall, ${ }^{2}$ Ryan Davis, 'Libby Augustine, 'Sarah Bright, ${ }^{1}$ Whitney Brown, ${ }^{1}$ Anneliese Harris, 'Sarah Holmes, 'Sayanthinie Jeyalingam, 'Sarah Large, ${ }^{1}$ Sylvia Mai, ${ }^{1}$ Eleanor Raper, ${ }^{1}$ Hang Wah Judtih Wong, ${ }^{1}$ Pok Man Wong, ${ }^{1,2}$ Carolyn M Sue, ${ }^{1}$ Barbara Lucas. ${ }^{1}$ Royal North Shore Hospital, St Leonards, NSW, Australia; ${ }^{2}$ Kolling Institute of Medical Research, St Leonards, NSW, Australia

\subsection{6/jnnp-2019-anzan.23}

Introduction Sporadic inclusion body myositis (sIBM) is the most common muscle disease affecting older adults with no disease-modifying treatment. Resistance exercises increase muscle hypertrophy, but weakness prevents exercising with higher resistance. In healthy subjects, augmentation of lightload training with blood flow restriction improved muscle strength $^{1}$; and similar exercises were safe in the elderly. ${ }^{2}$ We therefore investigate whether resistance exercises with blood flow restriction is safe and helpful in sIBM patients. We explored methods for exercising weak leg muscles, and options for better outcome measures.

Methods A matched-control pilot study, with 12-week treatment and 4-week follow-up periods, where participants concentrated on lower limbs resistance exercises with 50\% blood flow restriction 3 times/week, at 20\%-30\% of their repetition maximum. Patients are reviewed 4-weekly for muscle strength, blood biomarkers, 2-minute walk test (2MWT), timed up-andgo (TUG) test, minimal chair height standing ability test (MCHSAT), thigh girths, and quality of life scales.

Results 4 patients with varying abilities were on the exercise arm, and 3 acted as controls. Muscle groups with MRC score $\geq 2$ were able to be exercised. We found strength testing by hand-held dynamometer was unreliable, whereas the 2MWT, TUG test and MCHSAT showed less variability. All patients could perform their exercises at significantly increased repetitions or weights by week 4, without concerning adverse events, with trend towards continued improvement over the 4-month period.

Conclusion Resistance exercises with blood flow restriction appear safe, and may be helpful in sIBM patients, even in weakened muscles, enabling improvement in muscle strength and endurance.

\section{REFERENCES}

1. Hughes L, Paton B, Rosenblatt B, Gissane C, Patterson SD. Blood flow restriction training in clinical musculoskeletal rehabilitation: a systematic review and metaanalysis. Br J Sports 2017.

2. Vechin FC, Libardi CA, Conceicao MS, et al. Comparisons between low-intensity resistance training with blood flow restriction and high-intensity resistance training on quadriceps muscle mass and strength in elderly. J Strength Cond Res 2015.

\section{THE UTILITY OF NERVE BLOOD FLOW IN THE ASSESSMENT OF PERIPHERAL NEUROPATHY}

${ }^{1,2}$ Adeniyi Borire*, ${ }^{1}$ Tushar Issar, ${ }^{3}$ Natalie Kwai, ${ }^{4}$ Leo Visser, ${ }^{5}$ Ann Poynten, ${ }^{6}$ Neil Simon, ${ }^{7}$ Matthew Kiernan, ${ }^{1}$ Arun Krishnan. ${ }^{1}$ Prince of Wales Clinical School, UNSW, Sydney, NSW, Australia; ${ }^{2}$ Liverpool Hospital, Sydney, NSW, Australia; ${ }^{3}$ School of Medical Sciences, UNSW, Sydney, NSW, Australia; ${ }^{4}$ St Elisabeth Ziekenhuis, Tilburg, Netherlands; ${ }^{5}$ Department of Endocrinology, Prince of Wales Hospital, Sydney, NSW, Australia; ${ }^{6}$ St Vincent's Clinical School, University of New South Wales, Sydney, NSW, Australia; ${ }^{7}$ Brain and Mind Centre, Sydney, NSW, Australia

\subsection{6/jnnp-2019-anzan.24}

Introduction Animal and human studies of diabetes have shown significant alterations in nerve blood flow (NBF), which may also play a role in the development of neuropathy. However, the non-invasive assessment of NBF in human subjects has remained elusive until the recent technological advancements in ultrasound (US) technology. We undertook sonographic assessment of NBF in 75 patients with type 2 diabetes and correlated the findings with neuropathy severity scores and electrophysiological parameters.

Methods Blinded median and tibial nerve ultrasound scans were performed at non-entrapment sites using a high-resolution linear probe. NBF was quantified using power Doppler techniques to obtain the vessel score (VSc) and maximum perfusion intensity (MPI). Routine nerve conduction studies were performed, and neuropathy severity was assessed using the total neuropathy score (TNS). Aged- and gender-matched controls were enrolled.

Results Diabetic nerves had higher rates of NBF detection $(28 \%)$ compared to the control group $(p<0.0001)$. Significant correlations were found between NBF parameters and nerve size $(p<0.001)$, reported sensory symptoms $(p<0.05)$ and neuropathy severity scores $(\mathrm{p}<0.001)$. The cohort with diabetes had significantly larger median $\left(8.5 \pm 0.3 \mathrm{~mm}^{2}\right.$ vs. $7.2 \pm 0.1$ $\left.\mathrm{mm}^{2}, \mathrm{p}<0.05\right)$ and tibial $\left(18.0 \pm 0.9 \mathrm{~mm}^{2}\right.$ vs. $12.8 \pm 0.5$ $\left.\mathrm{mm}^{2}, \mathrm{p}<0.05\right)$ nerves compared to controls.

Conclusions Peripheral nerve hypervascularity is detectable by US in moderate to severe diabetic neuropathy with prominent sensory dysfunction. Consistent with previous studies, the sonographic detection of NBF is a pathological finding.

\section{VARIATION IN THE ANATOMY OF THE NORMAL HUMAN OPTIC CHIASM: AN MRI STUDY}

${ }^{1,2}$ Christian J Lueck* ${ }^{2}$ Nicholas Bosler, ${ }^{3}$ Andrew Neely, ${ }^{4}$ David Ashton. ${ }^{1}$ Department of Neurology, The Canberra Hospital, Canberra, ACT, Australia; ${ }^{2}$ Medical School, Australian National University, Canberra, ACT, Australia; ${ }^{3}$ School of Engineering, University of New South Wales, Canberra, ACT, Australia; ${ }^{4}$ Department of Radiology, Canberra Hospital, Canberra, ACT, Australia

\subsection{6/jnnp-2019-anzan.25}

Introduction Compression of the optic chiasm gives rise to bitemporal hemianopia. The reason for this is unclear, but one hypothesis suggests it relates to the fact that nasal retinal fibres cross each other while temporal fibres do not. This 'crossing' hypothesis has been investigated using finite element modelling but this requires accurate anatomical data. The precise shape of the chiasm is not clear: nasal fibres may not decussate centrally (as if the chiasm were ' $\mathrm{X}$ '-shaped) but, instead, decussate paracentrally and run 